\title{
ЧИСЛЕННАЯ МОДЕЛЬ ОСАЖДЕНИЯ ПОЛИДИСПЕРСНОЙ ТВЕРДОЙ КОМПОНЕНТЫ АЭРОЗОЛЯ НА ПЛАСТИНУ В ДВИЖУЩЕМСЯ ДОЗВУКОВОМ ВЫСОКОСКОРОСТНОМ ПОТОКЕ
}

\section{NUMERICAL MODEL OF DEPOSITION OF A POLYDISPERSE SOLID AEROSOL COMPONENT ON A PLATE IN A MOVING SUBSONIC HIGH-VELOCITY FLOW}

\section{Tukmakov}

Summary. The paper studies numerically the deposition of the solid component of aerosol in a moving stream of a dusty medium. To study the dynamics of an inhomogeneous medium, a polydisperse mathematical model of aerosol dynamics is used, which assumes different particle sizes and material densities of the dispersed phase fractions. When describing the motion of the dispersed component, the force of aerodynamic resistance of particles to the gas flow is taken into account. The model also allows one to take into account the effect of an electric field on aerosol particles.

Keywords: aerodynamics, multiphase media, numerical modeling, NavierStokes equation.

\section{Ввемение}

$\mathbf{M}$ ногие природные явления и промышленные технологии связаны с течениями неоднородных сред [1-15], в частности процессы, связанные с течением аэрозолей и запылённых сред встречаются В горной и химической промышленностях, аэрокосмических технологиях и т.д. В монографиях и публикациях в периодических изданиях изучаются вопросы около и сверхзвуковых течений в аэрозольных средах [2,3]. Исследуются течения в суспензиях, вызванные взаимодействием компонент смеси [4]. В ряде случаев представляет интерес исследование динамики неоднородных сред, находящихся под влиянием как аэродинамических, так и электрических полей $[5,6]$. Так как встречающиеся в технических приложениях потоки газокапельных и запыленных сред имеют не однородную дисперсную компоненту -частицы или капли могут отличаться размером и плотностью материала. То возникает необходимость в применении полидисперсной математической модели динамики газовзвесей (взвешенных в газе частиц). Данная работа посвящена численной модели динамики неоднородной электрически заряженной среды (аэрозоля) позволяющей выявить закономерности осаждения
Тукмаков Дмитрий Алексеевич

К.ф.-м.н., н.с., ФИЦ «Казанский научный чентр Российской академии наук», г. Казань tukmakovda@imm.knc.ru

Аннотация. В работе численно исследуется осаждение твердой компоненты аэрозоля в движущемся потоке запылённой среды. Для исследования динамики неоднородной среды применяется полидисперсная математическая модель динамики аэрозоля, предполагающая различные размеры частиц и плотности материала фракций дисперсной фазы. При описании движения дисперсной компоненты учитывается сила аэродинамического сопротивления частиц потоку газа. Также модель позволяет учесть воздействие электрического поля на частицы аэрозоля.

Ключевые слова: аэродинамика, многофазные среды, численное моделирование, уравнение Навье-Стокса.

фракций полидисперсной газовзвеси, движущейся в канале, на пластину к которой приложен электрический потенциал.

\section{Математическая \\ Mo $\triangle \mathrm{e} \wedge \mathrm{b}$}

Динамика полидисперсной газовзвеси описывается системой уравнений полидисперсной газовзвеси с учетом межфазного обмена импульсом и энергией. Движение несущей среды описывается системой уравнений Навье-Стокса [13,14] для сжимаемого теплопроводного газа с учетом межфазного силового взаимодействия и теплообмена:

$$
\begin{aligned}
& \frac{\partial \rho_{i}}{\partial t}+\nabla\left(\rho_{i} \mathbf{V}_{i}\right)=0,(i=1, \ldots n) \\
& \frac{\partial \rho_{1} V_{1}^{k}}{\partial t}+\nabla^{i}\left(\rho_{1} V_{1}^{k} V_{1}^{i}+\delta_{i k} p-\tau_{i k}\right)=-\sum_{j=2}^{m} F_{j k}+\sum_{j=2}^{m} \alpha_{j} \nabla^{k} p, \\
& (i, k=1,2), \\
& \frac{\partial \rho_{j} V_{j}^{k}}{\partial t}+\nabla^{i}\left(\rho_{j} V_{j}^{i} V_{j}^{k}\right)=F_{j k}-\alpha_{j} \nabla^{k} p,(j=2, \ldots n, i, k=1,2),
\end{aligned}
$$




$$
\begin{aligned}
& \frac{\partial\left(e_{1}\right)}{\partial t}+\nabla^{i}\left(V_{1}^{i}\left(e_{1}+p-\tau_{i i}\right)-V_{1}^{k} \tau_{k i}-\lambda \nabla^{i} T\right)= \\
& =-\sum_{j=2}^{n} Q_{j}-\sum_{j=2}^{n}\left|F_{j k}\right|\left(V_{1}^{k}-V_{j}^{k}\right)+\left(\sum_{j=2}^{n} \alpha_{j}\right) \nabla^{k}\left(p V_{1}^{k}\right) \\
& \frac{\partial\left(e_{j}\right)}{\partial t}+\nabla^{k}\left(e_{j} V_{j}^{k}\right)=Q_{j},(j=2, \ldots n, k=1,2), \\
& p=(\gamma-1)\left(e_{1}-\rho_{1}\left(u_{1}^{2}+v_{1}^{2}\right) / 2\right), \quad e_{1}=\rho_{1} I+\rho_{1}\left(u_{1}^{2}+v_{1}^{2}\right) / 2 \\
& \rho_{i}=\alpha_{i} \rho_{i 0}, e_{i}=\rho_{i} C_{p i} T_{i}
\end{aligned}
$$

Здесь $\boldsymbol{V}_{i}=\left[u_{i}, v_{i}\right]$-вектор скорости компонент смеси, тіj -тензор вязких напряжений несущей компоненты:

$$
\begin{aligned}
& \tau_{11}=\mu\left(2 \frac{\partial u_{1}}{\partial x}-\frac{2}{3} D\right), \tau_{22}=\mu\left(2 \frac{\partial v_{1}}{\partial y}-\frac{2}{3} D\right), \\
& \tau_{12}=\mu\left(\frac{\partial u_{1}}{\partial y}+\frac{\partial v_{1}}{\partial x}\right), D=\frac{\partial u_{1}}{\partial x}+\frac{\partial v_{1}}{\partial y} .
\end{aligned}
$$

Одним из важных параметров динамики многофазных сред является объёмное содержание дисперсной фазы, отношение объема компоненты смеси к общему объёму смеси- $\alpha_{i}=V_{i} / V$, при этом физическая плотность материала дисперсной фазы представляется неизменной [1-3]. Уравнение (1) описывает непрерывность плотности несущей среды и средней плотности фракций дисперсной фазы $(i>1)$. Уравнения (2) и (3) описывают законы сохранения компонент вектора импульса несущей среды и фракций дисперсной фазы соответственно. Уравнения (4) и (5) описывают законы сохранения полной энергии газа и тепловой энергии фракций дисперсной фазы. Сила межфазного взаимодействия включает в себя силу аэродинамического сопротивления, силу Архимеда и силу присоединенных масс [1]. Здесь $p, \rho_{l}, u_{l}, v_{l}$ -давление, плотность, декартовы составляющие скорости несущей среды в направлении осей $x$ и $y$ соответственно; $T_{l}, e_{1}$-температура и полная энергия газа; $\alpha_{i}, \rho_{i}, T_{i}, C_{p}, e_{i}, u_{i}, v_{i}$-объемное содержание фракции дисперсной фазы, средняя плотность, физическая плотность, температура, теплоемкость, внутренняя энергия, декартовы составляющие скорости фракций дисперсной фазы, $F_{j k}$ - составляющие вектора силового взаимодействия фракций дисперсной фазы и несущей среды, $k=1,2$; $Q_{j}$ - тепловой поток между $j$-ой фракцией дисперсной фазы и несущей средой, $j=2 \ldots n,[1,2,12]$ :

$$
\begin{aligned}
& F_{x i}=\frac{3}{4} \frac{\alpha_{i}}{\left(2 r_{i}\right)} C_{d i} \rho_{1} \sqrt{\left(u_{1}-u_{i}\right)^{2}+\left(v_{1}-v_{i}\right)^{2}}\left(u_{1}-u_{i}\right)+ \\
& ++\alpha_{i} \rho_{1}\left(\frac{\partial u_{1}}{\partial t}+u_{1} \frac{\partial u_{1}}{\partial x}+v_{1} \frac{\partial u_{1}}{\partial y}\right)+
\end{aligned}
$$

$$
\begin{aligned}
& +0.5 \alpha_{i} \rho_{1}\left(\frac{\partial u_{1}}{\partial t}+u_{1} \frac{\partial u_{1}}{\partial x}+v_{1} \frac{\partial u_{1}}{\partial y}-\frac{\partial u_{i}}{\partial t}-u_{i} \frac{\partial u_{i}}{\partial x}-v_{i} \frac{\partial u_{i}}{\partial y}\right)- \\
& -q_{0} \rho_{i} \partial \varphi / \partial x \\
& F_{y i}=\frac{3}{4} \frac{\alpha_{i}}{\left(2 r_{i}\right)} C_{d} \rho_{1} \sqrt{\left(u_{1}-u_{i}\right)^{2}+\left(v_{1}-v_{i}\right)^{2}}\left(v_{1}-v_{i}\right)+ \\
& +\alpha_{i} \rho_{1}\left(\frac{\partial v_{1}}{\partial t}+u_{1} \frac{\partial v_{1}}{\partial x}+v_{1} \frac{\partial v_{1}}{\partial y}\right)+ \\
& +0.5 \alpha_{i} \rho_{1}\left(\frac{\partial v_{1}}{\partial t}+u_{1} \frac{\partial v_{1}}{\partial x}+v_{1} \frac{\partial v_{1}}{\partial y}-\frac{\partial v_{i}}{\partial t}-u_{i} \frac{\partial v_{i}}{\partial x}-v_{i} \frac{\partial v_{i}}{\partial y}\right)- \\
& -q_{0} \rho_{i} \partial \varphi / \partial y, \\
& C_{d i}=\frac{24}{\operatorname{Re}_{i 1}}+\frac{4}{\operatorname{Re}_{i 1}^{0.5}}+0.4, \quad M_{1 i}=\left|\bar{V}_{1}-\overline{V_{i}}\right| / c \\
& \operatorname{Re}_{1 i}=\rho_{1}\left|\bar{V}_{1}-\bar{V}_{i}\right| 2 r_{i} / \mu, \operatorname{Pr}=C_{p} \mu / \lambda, \\
& N u_{1 i}=2 \exp \left(-M_{1 i}\right)+0.459 \operatorname{Re}_{1 i}^{0.55} \operatorname{Pr}^{0.33} \\
& Q_{i}=6 N u_{1 i} \lambda\left(T_{l}-T_{i}\right) / d_{i}, i=2, . . n
\end{aligned}
$$

При моделировании течения аэрозоля для составляющих скорости несущей и дисперсной компонент смеси задавались однородные граничные условия Дирихле. Для остальных динамических функций на границе расчетной области задавались однородные граничные условия Неймана. Составляющие силы Кулона на единицу объема газовзвеси определяются через ее удельный заряд, объемную плотность твердой фазы и напряженность электрического поля. Потенциал электрического поля в расчетной области определяется из решения уравнения Пуассона [17]:

$$
\begin{aligned}
& \operatorname{div} \boldsymbol{E}=\frac{\rho_{э n}}{\varepsilon \varepsilon_{0}}, \boldsymbol{E}=-\bar{\nabla} \varphi, \Delta^{2} \varphi=-\frac{\rho_{\ni n}}{\varepsilon \varepsilon_{0}} \\
& \rho_{э n}=\sum_{j=2}^{n} \alpha_{j} \rho_{j 0} \cdot q_{0}=\sum_{j=2}^{n} \rho_{j} \cdot q_{0}, \varepsilon_{0}=\frac{10^{-9}}{36 \pi} \Phi / \mathrm{M}, \varepsilon=1 .
\end{aligned}
$$

где $q_{0}$ - удельный заряд единицы массы твердой фракции, $\varphi-$ потенциал электрического поля. Для уравнения Пуассона задавались однородные граничные условия Неймана в той части канала в которой располагался однородный газ и однородные граничные условия Дирихле, в той части канала в которой располагалась электрически заряженная дисперсная компонента смеси. Система уравнений динамики многофазной среды (1)-(5) решалась явным конечноразностным методом Мак-Кормака [17]. Шаг по времени вычислялся, исходя из условия Куранта -Фридрихса Леви [14]. Рассмотрим применение численного метода на примере скалярного нелинейного уравнения в частных производных (7): 


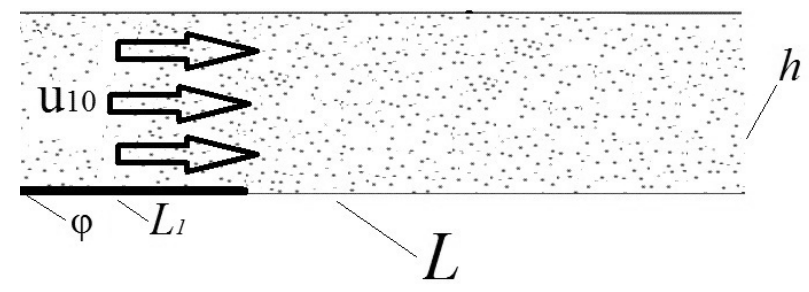

Рис. 1. Схематичное изображение канала с текущей по нему запылённой средой

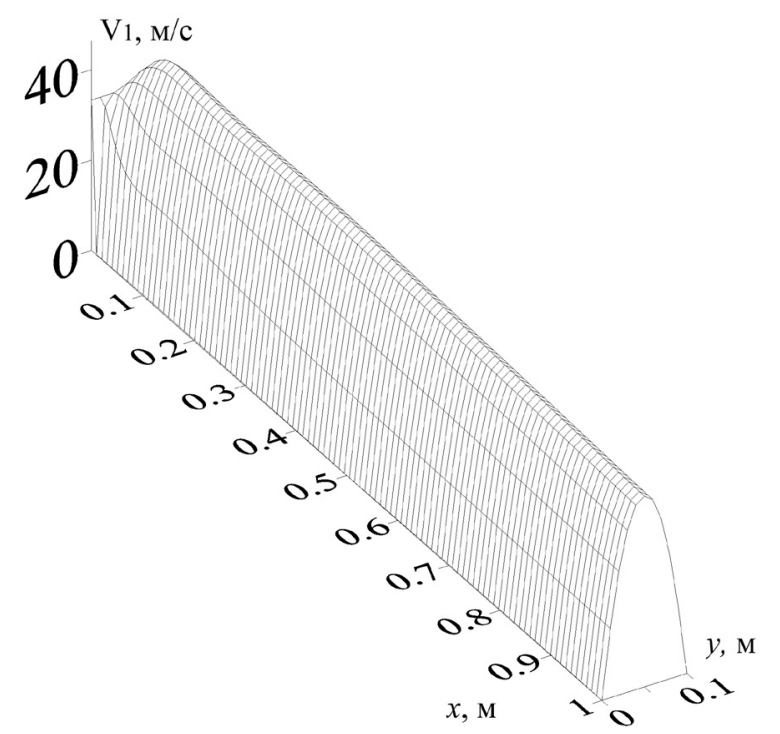

Рис. 2. Пространственное распределение модуля скорости газа в канале

$$
\frac{\partial f}{\partial t}+\frac{\partial a(f)}{\partial x_{1}}+\frac{\partial b(f)}{\partial x_{2}}=c(f)
$$

Алгоритм явного конечно-разностного метода МакКормака для нелинейного уравнения (7) имеет вид (8)(9):

$$
\begin{aligned}
& f_{j, k}^{*}=f_{j, k}^{n}-\frac{\Delta t}{\Delta x_{1}}\left(a_{j+1, k}^{n}-a_{j, k}^{n}\right)-\frac{\Delta t}{\Delta x_{2}}\left(b_{j, k+1}^{n}-b_{j, k}^{n}\right)+\Delta t c_{j, k}^{n} \\
& f_{j, k}^{n+1}=0,5\left(f_{j, k}^{n}+f_{j, k}^{*}\right)-0,5 \frac{\Delta t}{\Delta x_{1}}\left(a_{j, k}^{*}-a_{j-1, k}^{*}\right)- \\
& -0,5 \frac{\Delta t}{\Delta x_{2}}\left(b_{j, k}^{*}-b_{j, k-1}^{*}\right)+0.5 \Delta t c_{j, k}^{*}
\end{aligned}
$$

Здесь $\Delta x_{i}-$ шаг по соответствующему пространственному направлению, $\Delta t$ - шаг по времени. Для получения монотонного численного решения к сеточной функции на каждом временном шаге применялась схема нелинейной коррекции [15]. Алгоритм коррекции выполнялся последовательно вдоль всех узлов. Рассмотрим алгоритм коррекции решения на примере функции $f$. В случае если выполняются условия $\left(\delta f_{j-1 / 2} \cdot \delta f_{j+1 / 2}\right)<0 \quad$ или

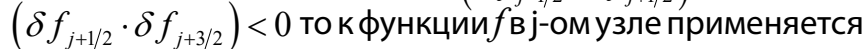
алгоритм схемы коррекции:

$$
\tilde{f}_{j}=f_{j}+\kappa\left(\delta f_{j+1 / 2}-\delta f_{j-1 / 2}\right)
$$

Нижний индекс обозначает номер узла сетки. Здесь использованы обозначения:

$$
\delta f_{j-1 / 2}=f_{j}-f_{j-1}, \delta f_{j+1 / 2}=f_{j+1}-f_{j}, \delta f_{j+3 / 2}=f_{j+2}-f_{j+1},
$$

в противном случае: $\tilde{f}_{i}=f_{i}, \tilde{f}_{j}$-значение функции в $j$-ом узле после перехода на $(n+1)$-ый временной слой по схеме Мак-Кормака, к- коэффициент коррекции. Для уравнения описывающего внутреннее электрическое поле дисперсной компоненты газовзвеси задавались однородные граничные условия Дирихле, в той части канала, в которой была расположена многофазная среда. И однородные граничные условия Неймана, в той части моделируемой области, которая предполагалась заполненной однородным газом. Уравнение Пуассона (6) интегрировалось методом установления [18].

\section{Результаты расчетов}

На рис. 1 схематично изображен канал с текущей по нему газовзвесью, на верхней и нижней стенке которого находятся электроды. Общая протяженность канала составляет- $L=1$ м. Ширина канала $h=0.1$ м. Длины электрода, находящегося на стенке канала, $L_{l}=0.1 \mathrm{M}$. 


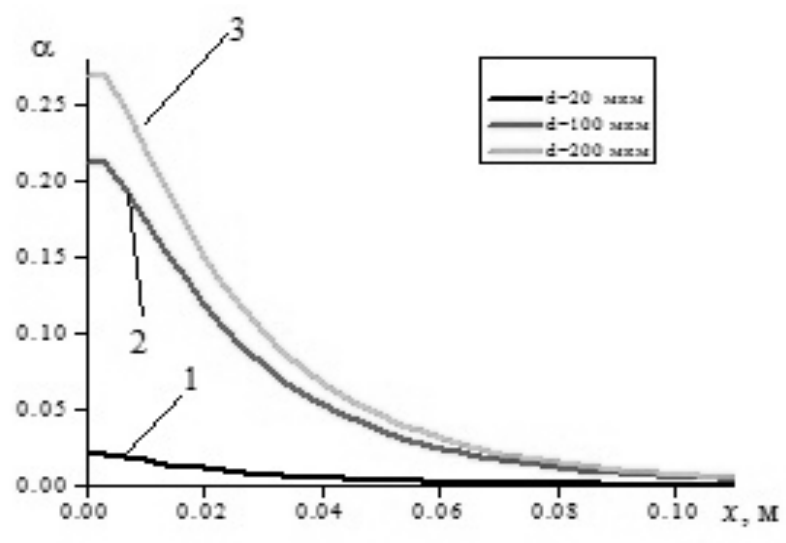

a

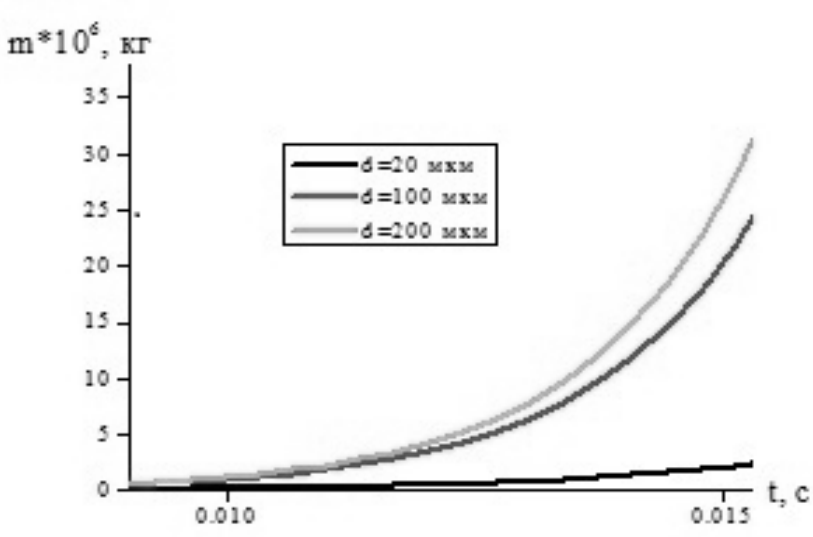

6

Рис. 3. Пространственное распределение объемного содержания фракций дисперсной фазы с плотностью материала $\rho 20=1850$ кг/м3 вблизи пластины - электрода, (y=0.002 м, момент времени $\mathrm{t}=5$ мс) -рис.3, а. Временная зависимость массы фракций дисперсной фазы осажденной на пластинуэлектрод-рис.3, 6.

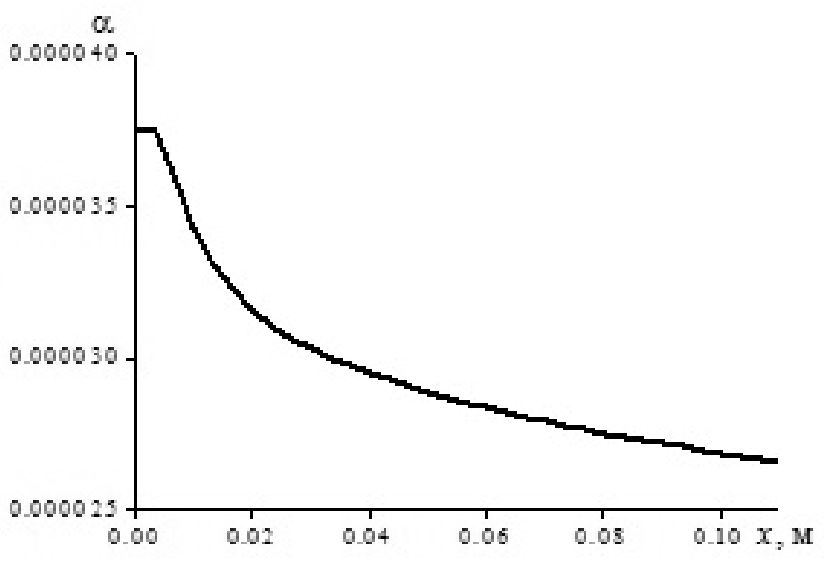

a

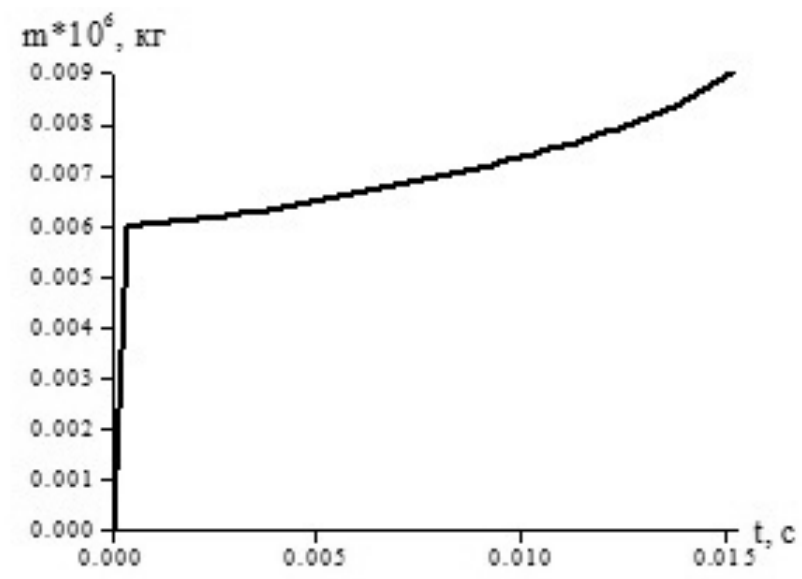

6

Рис. 4. Пространственное распределение объемного содержания фракции дисперсной фазы с плотностью материала $220=1850$ кг/м3 и диаметром частиц d=2 мкм вблизи пластины- электрода, ( $\mathrm{y}=0.002$ м, момент времени $\mathrm{t}=5$ мс) -рис.4, а. Временная зависимость массы фракции дисперсной фазы с диаметром частиц d=2 мкм осажденной на пластину-электрод-рис.4, 6

Скорость втекающего в канал газа составляла $u_{10}=40 \mathrm{M} / \mathrm{C}$, таким образом, величина числа Рейнольдса для данного

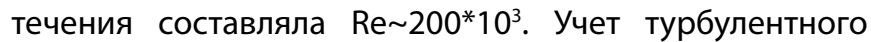
характера течения газа осуществлялся решением полной системы уравнений Навье-Стокса.

Удельный массовый заряд дисперсной фазы составляет $q_{0}=10^{-4}$ Кл/кг. Общее объёмное содержание дисперсной фазы описывается следующим выражением:

$$
\alpha=\sum_{j=2}^{m=6} \alpha_{j}=0.0001, \alpha_{2}=\ldots . .=\alpha_{6}=0.00002 \text {. }
$$

Потенциалы электрического поля на пластине: $\varphi=-5000$ В.Полученное численными расчётами пространственное распределение модуля скорости несущей среды в канале представлено на рис. 2. Наибольшего значения модуль скорости газа -

$$
V_{1}=\sqrt{u_{1}^{2}+v_{1}^{2}}
$$

достигает на оси симметрии канала.

Рассмотрим влияние дисперсности частиц на процесс осаждения дисперсной фазы газовзвеси 


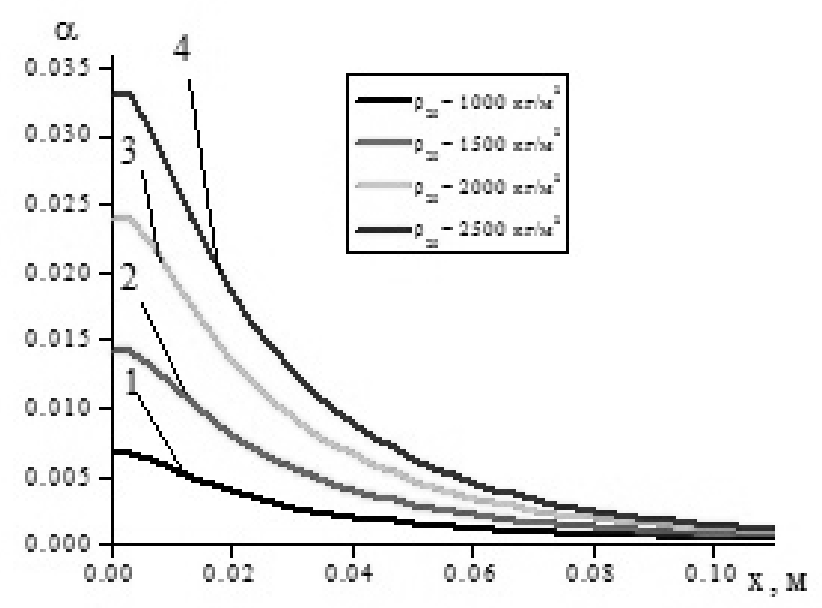

a

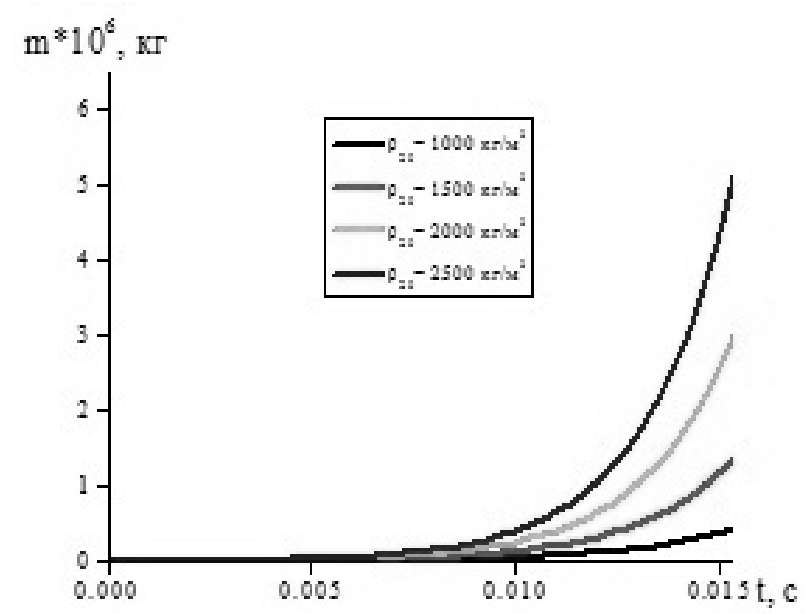

6

Рис. 5. Пространственное распределение объемного содержания фракций дисперсной фазы с диаметром частиц d=20 мкм вблизи пластины - электрода, ( $\mathrm{y}=0.002 \mathrm{~m}$, момент времени $\mathrm{t}=5 \mathrm{Mc})$ -рис.5, а. Временная зависимость массы фракций дисперсной фазы с диаметром частиц d=20 мкм осажденных на пластину-электрод-рис.5, 6

на поверхности электрода. На рис. 3(а, б) представлены результаты численных расчётов осаждения на пластину фракций твердой фазы запылённой среды в полидисперсной газовзвеси с одинаковой физической плотностью материала дисперсной фазы ( $20=1850 \mathrm{kг} /$ м3 -плотность бериллия) и различными размерами частиц. На рисунке 3 , а представлены пространственные распределения объемного содержания фракций газовзвеси вдоль координаты $\mathrm{x}(\mathrm{y}=0.002)$. Увеличение объемного содержания фракций дисперсной фазы газовзвеси вблизи пластины -электрода, происходит пропорционально размеру частиц. Временная зависимость массы осажденных на пластине-электроде частиц для различных фракций дисперсной фазы, также демонстрирует увеличение интенсивности напыления при увеличении размера частиц. Осаждение на пластине -электроде мелкодисперсных частиц происходит существенно менее интенсивно-рис.4 (а, б). Выявленные закономерности можно связать с тем, что увеличение размера частиц дисперсной фракции приводит к увеличению площади соприкосновения частицы и газа в квадратичной пропорции, тогда как масса частицы увеличивается в кубической пропорции. В моделируемом процессе сила Кулона, приложенная к частице, пропорциональна массе частицы, то есть увеличивается в кубической пропорции при увеличении размера частицы.

Численные расчеты демонстрируют влияние плотности материала вещества электрически заряженной запылённой среды на процесс напыления фракций дисперсной фазы газовзвеси на электрод- рис.5 (a, б). При одинаковом размере частиц (d=20 мкм) фракции с более плотным материалом дисперсных включений имеют большее объёмное содержание вблизи электрода ( $(y=0.002)$-рис.5, а. Также можно отметить, что фракции частиц с более плотным материалом имеют большею скорость напыления на поверхность электрода -рис.5, 6 .

Выявленные особенности осаждения на поверхность пластины полидисперсной газовзвеси, связаны с тем, что при одинаковом размере частиц более плотные частицы имеют большею массу, по этой причине такие частицы испытывают большее воздействие силы Кулона при одинаковой площади взаимодействия с газом.

\section{Выво $ы$}

В работе численно моделировался процесс осаждения на пластину-электрод электрически заряженной полидисперсной запылённой среды. Математическая модель учитывает вязкость несущей среды, воздействие на дисперсную компоненту смеси различных аэродинамических сил и силы Кулона, а также полидисперсный состав твердой компоненты аэрозоля. Было выявлено влияние таких свойств дисперсной фазы аэрозоля, как плотность материала частиц и их размер, на процесс осаждения фракций дисперсной фазы двухкомпонентной смеси. Численное моделирование продемонстрировало большую скорость осаждения частиц более крупных размеров. При одинаковом размере частиц, более интенсивно осаждаются частицы с большей плотностью материала. 
Мелкодисперсные частицы более подвержены воздействию аэродинамических сил, по этой причине сносятся потоком газа и имеют существенно меньшею скорость осаждения на пластину-электрод находящеюся на стенке канала, тогда как динамика крупнодисперсных частиц в большей степени определяется электрическим полем. Выявленные закономерности осаждения полидисперсного аэрозоля в движущемся потоке возможно использовать как при оптимизации технологий нанесения дисперсных покрытий на напыляемые поверхности, так и при оптимизации технологий очистки промышленных выбросов.

\section{ЛИТЕРАТУРА}

1. Нигматулин Р. И. Основы механики гетерогенных сред. Москва: Наука, 1978. - 336 с.

2. Кутушев А. Г. Математическое моделирование волновых процессов в аэродисперсных и порошкообразных средах. Санкт-Петербург: «Недра», $2003 .-284$.

3. Федоров А.В., Фомин В. М., Хмель Т. А. Волновые процессы в газовзвесях частиц металлов. Новосибирск, 2015.-301 с.

4. Невский Ю.А., Осипцов А. Н. Медленная гравитационная конвекция дисперсных систем в областях с наклонными границами //Известия Российской академии наук. Механика жидкости и газа. 2011. № 2. С. 65-81.

5. Дикалюк А.С., Суржиков С.Т. Численное моделирование разреженной пылевой плазмы в нормальном тлеющем разряде// Теплофизика высоких температур. 2012. Т. 50, № 5. С. 611-619.

6. Семенов В.П., Тимофеев А. В. Параметрический резонанс и перенос энергии в пылевой плазме//Математическое моделирование. 2018. Т. 30. № 2. С. 3-17.

7. Tadaa Y., Yoshioka S., Takimoto A., Hayashi Y. Heat transfer enhancement in a gas-solid suspension flow by applying electric field// International Journal of Heat and Mass Transfer. 2016. Volume 93. P. 778-787.

8. Тукмаков Д. А. Конечно-разностная модель динамики гомогенной смеси в применении к исследованию распространения и отражения ударной волны большой интенсивности в водородно-воздушной среде // Модели, системы, сети в экономике, технике, природе и обществе. 2020. № 1. С. 86-97.

9. Тукмаков Д. А. Математическая модель нестационарной сорбции в двухфазной среде, учитывающая пространственную неравномерность распределения концентрации микрокомпонента в фазе сорбента // Вестник Тверского государственного университета. Серия «Химия». 2019. № 4. С. $26-35$.

10. Тукмаков Д. А. Численное исследование скоростного скольжения фаз при прохождении ударной волны малой интенсивности из чистого газа в запылённую среду // Многофазные системы, 2019, том 14, № 2, С. 125-131.

11. Тукмаков А.Л., Тукмаков Д. А. Генерация акустического возмущения движущейся заряженной газовзвесью// Инженерно-физический журнал, 2018, № 5 , C. 1207-1213.

12. Тукмаков А.Л., Баянов Р.И, Тукмаков Д. А. Течение полидисперсной газовзвеси в канале, сопровождающееся коагуляцией в нелинейном волновом поле. Теплофизика и аэромеханика. 2015. Т. 22. № 3. С. 319-325.

13. Лойцянский Л. Г. Механика жидкости и газа. Москва: Издательство “Дрофа”, 2003. -784 с.

14. Fletcher C. A. Computation Techniques for Fluid Dynamics. Berlin: Springer-Verlang, 1988. -502 p.

15. Музафаров И.Ф.,Утюжников С. В. Применение компактных разностных схем кисследованию нестационарных течений сжимаемого газа // Математическое моделирование. 1993. Т.5. № 3. С.74-83.

16. Тукмаков А.Л. Численное моделирование акустических течений при резонансных колебаниях газа в закрытой трубе // Известия высших учебных заведений. Авиационная техника. 2006. № 4. С. 33-36

17. Сальянов Ф. А. Основы физики низкотемпературной плазмы, плазменных аппаратов и технологий. Москва: Наука, 1997.-240 с.

18. Крылов В.И., Бобков В. В., Монастырный П. И. Вычислительные методы. Т. 2, Москва: «Наука», 1977.—401 с.

(c) Тукмаков Дмитрий Алексеевич ( tukmakovda@imm.knc.ru ).

Журнал «Современная наука: актуальные проблемы теории и практики» 